\title{
Update on HHV-8-Associated Malignancies
}

\author{
Meena Sunil • Erin Reid • Mary Jo Lechowicz
}

Published online: 26 March 2010

(C) The Author(s) 2010. This article is published with open access at Springerlink.com

\begin{abstract}
The human herpesvirus 8 (HHV-8) is the oncogenic virus associated with Kaposi's sarcoma (KS) and lymphoproliferative disorders, namely, primary effusion lymphoma and multicentric Castleman's disease. KS is among the most common malignancies seen in HIVinfected patients despite the decreased incidence of KS in the era of highly active antiretroviral therapy. Advances in molecular pathology reveal HHV-8 tumorigenesis is mediated through molecular mimicry wherein viral-encoded proteins can activate several cellular signaling cascades while evading immune surveillance. This knowledge has led to the evolution of multiple therapeutic strategies against specific molecular targets. Many such therapeutic modalities have shown activity, but none have proven to be curative. Identifying possible prognostic factors is another active area of research. This review summarizes the recent developments in the fields of virus transmission, molecular biology, and treatment of HHV-8-related neoplasms.
\end{abstract}

Keywords Human herpesvirus $8 \cdot \mathrm{HHV}-8 \cdot \mathrm{KSHV}$.

Kaposi's sarcoma $\cdot$ Primary effusion lymphoma .

Multicentric Castleman's disease

M. Sunil • M. J. Lechowicz $(\bowtie)$

Department of Hematology/Medical Oncology,

Winship Cancer Institute, Emory University,

Room 2054, 1365C Clifton Road,

Atlanta, GA 30322, USA

e-mail: mlechow@emory.edu

M. Sunil

e-mail: msunil@emory.edu

E. Reid

Hematology/Oncology, Moores Cancer Center,

University of California, San Diego,

3855 Health Sciences, MC \#0987 La Jolla, CA, USA

e-mail: egreid@ucsd.edu

\section{Introduction}

Viruses have been linked to several human cancers since the latter half of the 20th century. This finding is especially so in immunosuppressed individuals. One of the harbingers of the HIV/AIDS epidemic in the 1980s was the sudden surge in the incidence of Kaposi sarcoma (KS) among male homosexuals [1]. Beral et al. [2] in 1990 proposed a sexually transmitted infectious agent could be the etiology of AIDS-related KS; however, it was not until 1994 that Chang et al. [3] identified the virus from KS tissues in a patient with AIDS and established its association with KS. This new $\gamma$ herpesvirus was named KS-associated herpesvirus (KSHV)/human herpesvirus-8 (HHV-8). HHV-8 is one of several oncogenic viruses. In addition to KS, HHV8 is associated with lymphoproliferative disorders including multicentric Castleman's disease (MCD), plasmablastic lymphoma, primary effusion lymphoma (PEL), and the solid variant of PEL [4-6]. A germinotropic lymphoproliferative disorder seen in HIV-seronegative patients is also linked to HHV-8 where the lesions are coinfected with Epstein-Barr virus (EBV) [7]. Deviations from these classic forms of HHV-8-associated malignancies have been reported, such as a case of large B-cell lymphoma coinfected with HHV-8 and EBV having histologic (although not immunophenotypic) similarities to classic Hodgkin's lymphoma in an immunocompetent person and another case of HHV-8-associated intravascular large B-cell lymphoma in an HIV-positive patient [8].

\section{HHV-8 Transmission}

The definite route of transmission of HHV-8 is still debated. Pica and Volpi [9॰] reviewed the possible routes of HHV8 transmission, including horizontal, sexual, vertical, blood 
borne, and through organ transplantation. A large, populationbased, cross-sectional study evaluating the epidemiology of HHV-8 in the general US adult population demonstrated that HHV-8 transmission may be related to sexual activity in men, especially in male homosexuals, whereas evidence was lacking for heterosexual transmission in women [10 ]. HHV8 infection was found to occur with increased prevalence among patients with recently acquired HIV infection in Sao Paulo, Brazil [11]. Both univariate and multivariate analysis found positive correlation between HHV-8 transmission during the first year of HIV infection and sexual practices: in men who have sex with men (MSM) and in persons with history of other sexually transmitted diseases.

In Africa, many have postulated the high seroprevalence observed in adults implied HHV-8 infection was acquired in childhood; however, recent data do not support this. Butler et al. [12] found a low seroprevalence of HHV8 infection among children in South Africa, in contrast to Uganda, where an age-dependent pattern was observed (rates increased with age). This finding suggests the possibility of different patterns of transmission of HHV-8 in Africa, the continent with highest prevalence of HHV-8. A large prospective study conducted in Zambia in a cohort of children from birth to 48 months to estimate annual incidence of HHV-8 concluded that transmission in children is mainly horizontal, through maternal mastication of food. This study also found the presence of HIV-1 infection increases the risk for acquiring HHV-8 in areas highly endemic for both viruses [13].

Further HHV-8 transmission studies in the HIVseronegative population demonstrate additional data in the transmission, development, and persistence of HHV-8associated malignancy. Transmission of HHV8 has been documented in the setting of organ transplantation $[14,15]$. Postallograft medical immunosuppression is associated with increased risk of KS, as well as other viral-associated malignancies (EBV lymphoproliferative disease). In this setting, reduction or withdrawal of immunosuppression can result in regression of $\mathrm{KS}$, even in the absence of other specific therapy [15]. Similarly, immune reconstitution through use of highly active antiretroviral therapy (HAART) in persons with HIV can be associated with spontaneous regression of KS.

\section{Oncopathogenesis}

The past two decades have seen significant advances describing the molecular pathways involved in HHV-8induced malignancies. There appears to be an intricate interplay between the host immune system and the virus, which results in tumorigenesis with evasion of immune surveillance. For a more comprehensive review of HHV-8- induced oncogenesis, the reader is referred to Liang et al.

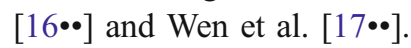

KSHV/HHV-8 displays a latent and a lytic phase. The latent phase, which is the default phase of HHV-8, helps with immune evasion and in establishing a persistent viral infection. The major latent viral proteins include latencyassociated nuclear antigen (LANA1), viral cyclin (v-cyc), and the viral Fas-associated death domain interleukin-1B converting enzyme (FLICE) inhibitory protein (vFLIP). Kaposin, viral interferon regulatory factors 3 (vIRF3), and KSHV-encoded microRNA (miRNA) are among the other latent viral proteins [16••]. Viral G-protein coupled receptor (vGPCR) and viral interleukin-6 (vIL-6) are lytic-phase proteins, although vIL- 6 is also often found in latently infected cells $[16 \bullet \bullet, 18 \cdot \bullet]$.

Although KSHV is thought to involve infection of endothelial/spindle cells in KS, it infects lymphocytes in both PEL and MCD. KSHV latent gene products, including LANA and v-FLIP, appear to dominate the malignant states of KS and PEL; the virus is considered to be predominantly in a latent state within both tumors. By contrast, in the setting of MCD, high levels of KSHV lytic gene productsincluding ORF5, ORF59, ORF65, and K8 - are typically found, particularly in the mantle zone region of the affected lymph nodes [19].

Functions and Oncogenicity of HHV-8 Viral Products

\section{LANA1}

This latent viral protein is essential to maintain latency and for cell proliferation. It may also have a role in immune evasion through inhibition of antigen presentation [20]. Transforming growth factor- $\beta$ (TGF- $\beta$ ) signaling pathway is an important regulator of growth in several cell types and also has a role in immune surveillance. KSHV LANA has been shown to downregulate TGF- $\beta$ type II receptor (TßRII) in KSHV-infected PEL cells through DNA methylation and deacetylation of proximal histones, blocking the TGF- $\beta$ signaling pathway and thus contributing to the neoplastic process [21]. LANA1 also inhibits the tumor suppressors P53 and von Hippel-Lindau (VHL) through its E3 ubiquitin ligase activity, which targets these suppressors for proteasome degradation. The consequences of this process include impaired apoptosis and increased hypoxiainducible factor- $1 \alpha$ levels, which leads to activation of genes involved in angiogenesis, cell proliferation, and survival [22].

$v-c y c$

V-cyc is a homologue of cellular D-type cyclins; it activates the DNA damage checkpoint, especially in early premalignant 
lesions, which would be expected to have protective effects against malignant transformation and persistence. However, the v-cyc may also lead to centrosomal abnormalities that could contribute to malignant transformation through genomic instability [23].

\section{$v F L I P$}

This latent viral protein has been associated with cell survival, morphologic change, and inflammatory activation. In addition, vFLIP appears to have a role in maintaining viral latency. Ye et al. [24] recently showed that vFLIP activation of the nuclear factor- $\mathrm{KB}(\mathrm{NF}-\mathrm{kB})$ was associated with suppression of the activator protein-1 (AP-1) pathway, which in turn inhibited the reactivation transcriptional activator (RTA) expression involved in KSHV lytic replication. However, this inhibition of lytic replication by NF- $\mathrm{KB}$ activation can be bypassed partly or overcome during a full lytic infection, and NF- $\mathrm{KB}$ activation and lytic gene expression are not mutually exclusive in all circumstances [24, 25]. Adding to the functional repertoire of vFLIP is a role in maintaining host-pathogen equilibrium together with IRF-1 through regulation of antigen presentation, according to the model proposed by Lagos et al. [26].

\section{$v G P C R$}

A lytic gene, $v G P C R$ is the only KSHV gene identified with transforming capacity. The $v G P C R$ gene can drive autocrine and paracrine Akt activation in infected endothelial cells. Also, $v G P C R$ unblocks a break on the mammalian target of rapamycin (mTOR) signaling pathway, which results in increased cell proliferation, cellular nutrient uptake, and angiogenesis [27].

\section{MicroRNA}

These are noncoding RNAs about 22 nucleotides in length; they function through interaction with messenger RNA, causing its degradation or preventing translation [28]. KSHV/HHV-8 encodes 12 microRNAs (miRNAs) coordinately expressed in the latency region. One of the viral miRNAs encoded by KSHV, miR-K12-11, appears to be homologous to cellular miR-155, and thus capable of using its binding sites [29, 30]. Also, miR-155 has a high expression in many human B-cell lymphomas, where it plays a significant role in differentiation, B-cell maturation, and regulation of the immune system, mediated via cytokine production [31]. Thus, viral miR-K12-11 and the oncogenic human miRNA, miR-155, potentially have the same target genes; this could possibly contribute to the pathogenesis of HHV-8-associated malignancies [29,
30]. Samols et al. [32] have revealed more cellular genes that are targeted by HHV-8 miRNAs, including thrombospondin 1 (THBS1), which is down regulated. THBS1 has a significant role in several cellular processes, including adhesion, migration, and angiogenesis [32].

\section{LANA2}

Another latent viral protein, LANA2 has a possible role in developing resistance to the drug paclitaxel by binding to the polymerized microtubules, decreasing their stability and interfering with the binding of the drug to the tubules [33].

\section{$O R F-K 1$}

The variations found in the highly variable glycoprotein, an early lytic-cycle gene product encoded by the ORF-K1 gene, defined four major subtypes and 13 variants or clades of HHV-8 [34]. These subtypes are variably distributed in different geographic areas of the world [34]. Subtypes A and $\mathrm{C}$ were seen mostly in the United States and Europe, whereas subtype B was almost exclusive to Africa [34, 35]. In a recent study of Italian patients with classic KS, HHV8 subtype A was shown to be associated with high blood levels of the virus, and these patients developed a rapidly progressive disease; thus, aggressive therapeutic strategies are required in persons infected with this subtype of HHV-8 [35].

Brown et al. [36] showed preliminary evidence linking host immunogenetic factors to control of HHV-8 infection. These investigators showed a high HHV-8 lytic antibody titer - a possible marker of lytic reactivation - was associated with genetic variations in cytokines in HIV-negative patients without KS.

\section{HHV-8-Associated Malignancies}

\section{Clinical Features and Therapeutic Options}

\section{Kaposi Sarcoma}

$\mathrm{KS}$ is an angioproliferative tumor and is among the most common malignancies seen in the HIV-infected population. The HIV/AIDS cancer match study by Engels et al. [37•] showed a greatly increased incidence of KS in the HIVinfected individuals compared with the general population (standardized incidence ratio 1300), and although incidence declined considerably in the HAART era, it still remains elevated in relation to the HIV-negative population. KS may involve the skin, lymph nodes, or viscera and is often multifocal. Compared with asymptomatic HHV-8 carriers, a significantly lower level of HHV-8-specific cytotoxic T cells has been noted in both AIDS-related and classic KS 
[38]. The iatrogenic KS associated with organ transplantation often regresses with reversal of immunosuppression or dose reduction of immunosuppressive agents. Similarly, improvement in the immune system brought on by use of HAART helps to alleviate HIV-related KS. There has been a notable sixfold drop in the incidence rate of KS from the pre-HAART era to the HAART era, supporting the hypothesis that immune impairment is permissive of $\mathrm{KS}$ [39]. A study showed that low CD4 counts and lack of antiretroviral therapy (ART) were major risk factors in KS development in the HHV-8-seropositive male homosexuals with AIDS [40•]. These investigators also demonstrated that HAART had a significant protective role not only with respect to prevention of KS but also in lowering mortality of patients with KS.

In the HAART era, there are increasing reports of KS occurring in HIV-infected individuals with suppressed HIV viral loads and apparent immune reconstitution. Maurer et al. [41] reported on nine HIV-infected patients with persistent cutaneous KS despite being on HAART, with CD4 counts greater than 300 cells $/ \mathrm{mm}^{3}$ and viral load less than 300 copies $/ \mathrm{mL}$ for nearly 2 years. Similar instances have been observed in the past, as noted by Krown et al. [42], which suggest the need to explore further the factors involved in development and progression of KS and to identify which patients respond to ART. A Swiss HIV cohort study followed 144 HIV-infected patients with KS from 1996 to 2004 with the aim of identifying adverse prognostic factors; T1 stage of tumor, CD4 count below 200 cells $/ \mu \mathrm{L}$, and a positive HHV-8 DNA in the plasma were associated with poorer outcomes [43]. In a retrospective cohort of 64 patients with KS who were treated with a combination of chemotherapy and HAART, the median time to initial response was as long as 9 months and the estimated time to complete resolution was 33 months [44]. The cumulative resolution probability at 3 years was $51 \%$. Although both HAART and chemotherapy were independently associated with initial clinical improvement, the authors found only recent HAART use significantly correlated with complete resolution of the disease. However, they failed to find any impact of CD4 counts and tumor stage on either improvement or resolution of lesions, although a low HIV viral load was a predictor of response to therapy. Furthermore, the type of HAART regimen used did not impact response significantly. The association of HHV-8 viral load with development of new lesions or with disease progression was again demonstrated in two recent studies [45, 46]. Stebbing et al. [47] conducted a prospective cohort study to develop an easily quantifiable prognostic index for patients with AIDS-related KS. Four prognostic factors were identified: age, KS occurring at or after AIDS onset, presence of comorbidities, and CD4 cell count.
Treatment options for KS include surgical removal, radiation therapy, and chemotherapy. Several standard chemotherapeutic agents have activity alone and in combination against KS: anthracyclines - most notably liposomal preparations, microtubule inhibitors, and vinca alkaloids. Recent advances in understanding the molecular pathogenesis of KS has lead to novel strategies targeting HHV-8.

Rapamycin (sirolimus), an mTOR inhibitor, has been shown to improve the levels of HHV-8-specific cytotoxic T cells when used in HHV-8-seropositive organ transplant recipients [48-50]. Sirolimus has been recommended as an immunosuppressive agent for organ transplant recipients in light of its beneficial effects in causing regression of KS and other posttransplant tumors without adversely impacting the graft [51]. Matrix metalloproteinases (MMP), the zinc-dependent endopeptidases, are overexpressed in KS cells and are involved in tumor invasion and metastasis. The AIDS Malignancy Consortium recently conducted a phase 2 trial comparing two doses $(50 \mathrm{mg}$ and $100 \mathrm{mg})$ of an MMP inhibitor known as COL-3 (CollaGenex Pharmaceuticals, Newtown, PA), a modified tetracycline, in AIDSrelated KS [52]. The overall response rate was $41 \%$ in the lower dose group and there were no serious adverse events, which raises the possibility of using this agent along with others for future therapy of AIDS-related KS. Another treatment approach is the induction of lytic viral proteins to render the virus more susceptible to the immune system and possibly enhance apoptosis or lysis of HHV-8-infected tumor cells. A recently published pilot trial using valproic acid was designed to determine the level of lytic expression of HHV-8 within the KS lesions [53]. The study results failed to show sufficiently high levels of lytic gene expression in a 30-day period on valproic acid. This therapeutic approach might still hold promise for the future, and additional studies using agents expected to be more potent inducers of lytic activation are underway.

\section{Multicentric Castleman's Disease}

HHV-8-associated MCD, an aggressive disease, is encountered mostly in immunosuppressed individuals, including HIV-infected patients. These patients are at risk of developing a plasmablastic lymphoma, a type of large Bcell lymphoma. For a comprehensive review of MCD in HIV-infected patients, the reader is referred to Stebbing et al. [54•]. In a histologic study of lymph nodes involved by HIV-associated MCD, as much as $63 \%$ of the lymph nodes tested positive for both MCD and KS, suggestive of coexistence of the two pathologic processes [55]. A rare instance of KSHV/HHV-8-associated hemophagocytic syndrome developing in an immunocompetent patient who also had coexistent MCD and KS was reported recently [56]. Wyplosz et al. [57] reported an instance of skin rash 
secondary to reactivation of HHV-8 with subsequent development of MCD as a continuous process. The authors further hypothesized that infected plasmablasts in the blood may be implicated in transport of the virus to the various target tissues as well as in the development of other HHV8 -associated conditions such as KS. Seliem et al. [58] reported another unusual variant of MCD-associated plasmablastic lymphoproliferative disorder, which had overlapping histologic features of plasmablastic microlymphoma and germinotropic lymphoproliferative disorder in an HIVpositive patient with HHV-8 and EBV coinfection.

Treatment for MCD has traditionally involved aggressive chemotherapy, and lately monoclonal antibodies against CD20 (rituximab) and IL-6 (atlizumab) have been effective $[18 \cdot \bullet$. A combination of rituximab with the immunomodulator thalidomide has been reported to cause regression of MCD in an HIV-infected patient [59]. A few recent trials have shown the beneficial effect of rituximab along with HAART in MCD with a prolonged resolution of symptoms. In the single-group, phase 2 trial by Bower et al. [60], the partial response was $67 \%$ and the 2-year overall survival (OS) was $95 \%$. In another prospective, open-label trial by Gerard et al. [61••], sustained remission at 1 year was achieved in $71 \%$, and OS at 1 year was $92 \%$.

\section{Primary Effusion Lymphoma}

PEL is a rare form of lymphoproliferative disorder seen frequently in HIV-infected patients; it presents as a classic and solid variant and generally has a very poor prognosis [62]. Reports exist of PEL occasionally occurring in HIVnegative patients, especially in the setting of other forms of immunosuppression (eg, in organ transplant recipients and in patients with chronic hepatitis B) [62-65]. Morphologically, it shares features of large-cell immunoblastic and anaplastic large-cell lymphoma [62, 66]. PEL tumor cells have a null phenotype, but are believed to be of B-cell origin [62]. Occasionally, the tumor cells may express Bcell or T-cell markers, which make detection of HHV-8 an important confirmatory test [66]. The level of IL-6 in these tumor cells is quite high, which aids in the diagnosis of PEL and could be a potential target for therapy [18••]. De Filippi et al. [67], in their report of three HIV-negative patients with hepatitis $C$ virus infection and PEL, showed elevated levels of free $\lambda$ light chains in the serum; levels correlated with clinical response to treatment. This finding can possibly be used to monitor response to therapy.

The rarity of PEL precludes any large, prospective trials and optimal therapy is lacking. Apart from the traditional chemotherapy, various regimens that have been tried include the proteasome inhibitor, bortezomib, which inhibits the NF-KB pathway, antivirals including cidofovir and ganciclovir, and the mTOR inhibitor rapamycin [18••].
Rapamycin decreases production of vascular endothelial growth factor (VEGF) as well as VEGF-induced signaling, thereby inhibiting accumulation of body fluids; this has clinical relevance in PEL therapy, but at the same time resistance to rapamycin developed quickly, limiting its efficacy [68]. Despite beneficial effects of rapamycin use in the HHV-8 malignancies in organ transplant patients, PEL has been reported to develop in renal transplant recipients who were on rapamycin, which might possibly suggest that this drug may not be as effective in preventing PEL as it is with KS [63]. Reports are mixed regarding the activity of bortezomib in PEL [69]. There have been recent case reports of long-term remission with HAART alone as therapy for PEL, as well as remission seen with radiotherapy in a patient with PEL who was refractory to chemotherapy $[70,71]$.

Recently, a randomized, controlled trial showed efficacy of the antiviral drug valganciclovir in decreasing HHV8 replication, which makes it useful as an adjunctive agent in HHV-8-associated disease processes, especially those with more lytic viral replication (eg, MCD) [72]. Another reported potential therapeutic approach included use of plant extracts, which were identified using a new fluorescence-based assay [73]. Last but not least, Bryant and Milliken [74] reported the successful treatment of PEL in an HIV-infected patient in second remission with allogeneic hemopoietic stem cell transplantation, who remained in complete remission at 31 months posttransplant only on HAART and with undetectable viral loads.

\section{Conclusions}

Our understanding of the pathophysiology and the molecular processes involved in the development of the various HHV-8-associated malignancies has increased dramatically over the past decade. Although many new targets have been identified at the molecular level for potential therapy of KS, $\mathrm{MCD}$, and PEL, therapeutic outcomes for these malignancies remain far from satisfactory. Future research should aim to improve our understanding of the role of host and viral factors that influence tumorigenesis and prognosis in the various HHV-8-related disease states, identifying the most efficacious combination from among the different therapeutic options through controlled trials. Alternative modalities, including radiotherapy and stem cell transplantation, should be explored in aggressive HHV-8 malignancies. Studies for effective prevention strategies, including vaccines, could potentially add to our arsenal against HHV-8.

Disclosure No potential conflict of interest relevant to this article was reported. 
Open Access This article is distributed under the terms of the Creative Commons Attribution Noncommercial License which permits any noncommercial use, distribution, and reproduction in any medium, provided the original author(s) and source are credited.

\section{References}

Papers of particular interest, published recently, have been highlighted as:

- Of importance

-. Of major importance

1. Hymes KB, Cheung T, Greene JB, et al.: Kaposi's sarcoma in homosexual men - a report of eight cases. Lancet 1981, 318:598600 .

2. Beral V, Peterman TA, Berkelman RL, et al.: Kaposi's sarcoma among persons with AIDS: a sexually transmitted infection? Lancet 1990, 335:123-128.

3. Chang Y, Cesarman E, Pessin MS, et al.: Identification of herpesvirus-like DNA sequences in AIDS-associated Kaposi's sarcoma. Science 1994, 266:1865-1869.

4. Cesarman E, Chang Y, Moore PS, et al.: Kaposi's sarcoma-associated herpesvirus-like DNA sequences in AIDS-related body-cavity-based lymphomas. N Engl J Med 1995, 332:1186-1191.

5. Soulier J, Grollet L, Oksenhendler E, et al.: Kaposi's sarcomaassociated herpesvirus-like DNA sequences in multicentric Castleman's disease. Blood 1995, 86:1276-1280.

6. Dupin N, Diss TL, Kellam P, et al.: HHV-8 is associated with a plasmablastic variant of Castleman disease that is linked to HHV8-positive plasmablastic lymphoma. Blood 2000, 95:1406-1412.

7. Du MQ, Diss TC, Liu H, et al.: KSHV- and EBV-associated germinotropic lymphoproliferative disorder. Blood 2002, 100:34153418.

8. Ferry JA, Sohani AR, Longtine JA, et al.: HHV8-positive, EBVpositive Hodgkin lymphoma-like large B-cell lymphoma and HHV8-positive intravascular large B-cell lymphoma. Mod Pathol 2009, 22:618-26.

9. - Pica F, Volpi A: Transmission of human herpesvirus 8: an update. Curr Opin Infect Dis 2007, 20:152-156. The review covers recent findings in the possible routes of $\mathrm{HHV}-8$ transmission. The interaction of host genetic factors and control of HHV-8 infection are briefly described.

10. • Engels EA, Atkinson JO, Graubard BI, et al.: Risk factors for human herpesvirus 8 infection among adults in the United States and evidence for sexual transmission. J Infect Dis 2007, 196: 199_ 207. This is the first reported population-based cross-sectional study to evaluate the epidemiology of HHV-8 in the general US adult population. This study was undertaken by the National Health and Nutrition Examination Survey III.

11. Batista MD, Ferreira S, Sauer MM, et al.: High human herpesvirus 8 (HHV-8) prevalence, clinical correlates and high incidence among recently HIV-1 infected subjects in Sao Paulo, Brazil. PLoS One 2009, 4:e5613.

12. Butler LM, Dorsey G, Hladik W, et al.: Kaposi sarcomaassociated herpesvirus (KSHV) seroprevalence in populationbased samples of African children: evidence for at least 2 patterns of KSHV transmission. J Infect Dis 2009, 200:430-438.

13. Minhas V, Crabtree KL, Chao A, et al.: Early childhood infection by human herpesvirus 8 in Zambia and the role of human immunodeficiency virus type 1 coinfection in a highly endemic area. Am J Epidemiol 2008, 168:311-320.
14. Luppi M, Barozzi P, Santagostino G, et al.: Molecular evidence of organ-related transmission of Kaposi sarcoma-associated herpesvirus or human herpesvirus-8 in transplant patients. Blood 2000, 96:32793281.

15. Einollahi B, Lessan-Pezeshki M, Nourbala MH, et al.: Kaposi's sarcoma following living donor kidney transplantation: review of 7,939 recipients. Int Urol Nephrol 2009, 41:679-685.

16. •- Liang C, Lee JS, Jung JU: Immune evasion in Kaposi's sarcoma-associated herpesvirus-associated oncogenesis. Semin Cancer Biol 2008, 18:423-436. This review provides insight into the interplay between HHV-8 and the host immune system. The role of HHV-8 in tumorigenesis and in evasion of immune surveillance is elaborated.

17. •- Wen KW, Damania B: Kaposi sarcoma-associated herpesvirus (KSHV): molecular biology and oncogenesis. Cancer Lett 2009 (Epub ahead of print). This review article provides an in-depth look at the virus biology and its oncopathogenesis. The therapeutic strategies for HHV-8-associated disorders are also briefly described.

18. •- Sullivan RJ, Pantanowitz L, Casper C, et al.: HIV/AIDS: epidemiology, pathophysiology, and treatment of Kaposi sarcomaassociated herpesvirus disease: Kaposi sarcoma, primary effusion lymphoma, and multicentric Castleman disease. Clin Infect Dis 2008, 47:1209-1215. The basics of epidemiology, virology, and immunologic aspects of HHV-8 and its associated neoplasms are discussed in this article. Recent findings and various molecular targets of therapy are highlighted.

19. Abe Y, Matsubara D, Gatanaga H, et al.: Distinct expression of Kaposi's sarcoma-associated herpesvirus encoded proteins in Kaposi's sarcoma and multicentric Castleman's disease. Pathol Int 2006, 56:617-624.

20. Zaldumbide A, Ossevoort M, Wiertz EJ, et al.: In cis inhibition of antigen processing by the latency-associated nuclear antigen I of Kaposi sarcoma herpesvirus. Mol Immunol 2007, 44:1352-1360.

21. Di Bartolo DL, Cannon M, Liu YF, et al.: KSHV LANA inhibits TGF-beta signaling through epigenetic silencing of the TGF-beta type II receptor. Blood 2008, 111:4731-4740.

22. Cai QL, Knight JS, Verma SC, et al.: EC5S ubiquitin complex is recruited by KSHV latent antigen LANA for degradation of the VHL and p53 tumor suppressors. PLoS Pathog 2006, 2:e116.

23. Koopal S, Furuhjelm JH, Jarviluoma A, et al.: Viral oncogeneinduced DNA damage response is activated in Kaposi sarcoma tumorigenesis. PLoS Pathog 2007, 3:1348-1360.

24. Ye FC, Zhou FC, Xie JP, et al.: Kaposi's sarcoma-associated herpesvirus latent gene vFLIP inhibits viral lytic replication through NF-kappaB-mediated suppression of the AP-1 pathway: a novel mechanism of virus control of latency. J Virol 2008, 82:4235-4249.

25. Grossman C, Ganem D: Effects of NFkappaB activation on KSHV latency and lytic reactivation are complex and contextdependent. Virology 2008, 375:94-102.

26. Lagos D, Trotter MW, Vart RJ, et al.: Kaposi sarcoma herpesvirusencoded vFLIP and vIRF1 regulate antigen presentation in lymphatic endothelial cells. Blood 2007, 109:1550-1558.

27. Sodhi A, Chaisuparat R, Hu J, et al.: TSC $2 / m T O R$ pathway drives endothelial cell transformation induced by the Kaposi's sarcomaassociated herpesvirus $\mathrm{G}$ protein-coupled receptor. Cancer Cell 2006, 10:133-143.

28. Cullen BR: Viruses and microRNAs. Nat Genet 2006, 38(Suppl): S25-S30.

29. Skalsky RL, Samols MA, Plaisance KB, et al.: Kaposi's sarcomaassociated herpesvirus encodes an ortholog of miR-155. J Virol 2007, 81:12836-12845.

30. Gottwein E, Mukherjee N, Sachse C, et al.: A viral microRNA functions as an orthologue of cellular miR-155. Nature 2007, 450:1096-1099. 
31. Thai TH, Calado DP, Casola S, et al.: Regulation of the germinal center response by microRNA-155. Science 2007, 316:604-608.

32. Samols MA, Skalsky RL, Maldonado AM, et al.: Identification of cellular genes targeted by KSHV-encoded microRNAs. PLoS Pathog 2007, 3:e65.

33. Munoz-Fontela C, Marcos-Villar L, Hernandez F, et al.: Induction of paclitaxel resistance by the kaposi's sarcoma-associated herpesvirus latent protein LANA2. J Virol 2008, 82:15181525.

34. Zong JC, Ciufo DM, Alcendor DJ, et al. High-level variability in the ORF-K1 membrane protein gene at the left end of the Kaposi's sarcoma-associated herpesvirus genome defines four major virus subtypes and multiple variants or clades in different human populations. J Virol 1999, 73:4156-4170.

35. Mancuso R, Biffi R, Valli M, et al.: HHV8 a subtype is associated with rapidly evolving classic Kaposi's sarcoma. J Med Virol 2008, 80:2153-2160.

36. Brown EE, Fallin MD, Goedert JJ, et al.: Host immunogenetics and control of human herpesvirus-8 infection. J Infect Dis 2006, 193:1054-1062.

37. • Engels EA, Biggar RJ, Hall HI, et al.: Cancer risk in people infected with human immunodeficiency virus in the United States. Int J Cancer 2008, 123:187-194. This study highlights the persistence of KS in the HAART era in HIV-infected patients. The study evaluates the risk of malignancy in the moderately immunosuppressed HIV population.

38. Guihot A, Dupin N, Marcelin AG, et al.: Low T-cell responses to human herpesvirus 8 in patients with AIDS-related and classic Kaposi sarcoma. J Infect Dis 2006, 194:1078-1088.

39. National Cancer Institute: SEER Cancer statistics review 19752004. Available at http://seer.cancer.gov/csr/1975 2004/results merged/sect_10_kaposi_sarcoma.pdf Accessed November 2009.

40. - Martro E, Esteve A, Schulz TF, et al.: Risk factors for human herpesvirus 8 infection and AIDS-associated Kaposi's sarcoma among men who have sex with men in a European multicenter study. Int J Cancer 2007, 120:1129-1135. This European multicenter study shows evidence of high prevalence of $\mathrm{HHV}$ 8 seropositivity among MSM. Low CD4 counts and lack of HAART were shown to be significant risk factors in development of $K S$.

41. Maurer T, Ponte M, Leslie K: HIV-associated Kaposi's sarcoma with a high CD4 count and a low viral load. N Engl J Med 2007, 357:1352-1353.

42. Krown SE, Lee JY, Dittmer DP: More on HIV-associated Kaposi's sarcoma. N Engl J Med 2008, 358:535-536.

43. El Amari EB, Toutous-Trellu L, Gayet-Ageron A, et al.: Predicting the evolution of Kaposi sarcoma, in the highly active antiretroviral therapy era. AIDS 2008, 22:1019-1028.

44. Nguyen HQ, Magaret AS, Kitahata MM, et al.: Persistent Kaposi sarcoma in the era of highly active antiretroviral therapy: characterizing the predictors of clinical response. AIDS 2008, 22:937-945.

45. Laney AS, Cannon MJ, Jaffe HW, et al.: Human herpesvirus 8 presence and viral load are associated with the progression of AIDS-associated Kaposi's sarcoma. AIDS 2007, 21:1541-1545.

46. Nsubuga MM, Biggar RJ, Combs S, et al.: Human herpesvirus 8 load and progression of AIDS-related Kaposi sarcoma lesions. Cancer Lett 2008, 263:182-188.

47. Stebbing J, Sanitt A, Nelson M, et al.: A prognostic index for AIDS-associated Kaposi's sarcoma in the era of highly active antiretroviral therapy. Lancet 2006, 367:1495-1502.

48. Stallone G, Schena A, Infante B, et al.: Sirolimus for Kaposi's sarcoma in renal-transplant recepients. N Engl J Med 2005, 352:1317-1323.
49. Barozzi P, Bonini C, Potenza L, et al.: Changes in the immune responses against human herpesvirus- 8 in the disease course of posttransplant Kaposi sarcoma. Transplantation 2008, 86:738744.

50. Ho CM, Huang SF, Hu RH, et al.: Sirolimus-induced signaling modifications in Kaposi's sarcoma with resolution in a liver transplant recipient. Clin Transplant 2009 (Epub ahead of print).

51. Monaco AP: The role of mTOR inhibitors in the management of posttransplant malignancy. Transplantation 2009, 87:157-163.

52. Dezube BJ, Krown SE, Lee JY, et al.: Randomized phase II trial of matrix metalloproteinase inhibitor COL-3 in AIDS-related Kaposi's sarcoma: an AIDS Malignancy Consortium Study. J Clin Oncol 2006, 24:1389-1394.

53. Lechowicz M, Dittmer DP, Lee JY, et al.: Molecular and clinical assessment in the treatment of AIDS Kaposi sarcoma with valproic acid. Clin Infect Dis 2009 (Epub ahead of print).

54. - Stebbing J, Pantanowitz L, Dayyani F, et al.: HIV-associated multicentric Castleman's disease. Am J Hematol 2008, 83:498503. This article presents a comprehensive review of the pathogenesis, presentation, and treatment options of the rare lymphoproliferative disorder, $M C D$.

55. Naresh KN, Rice AJ, Bower M: Lymph nodes involved by multicentric Castleman disease among HIV-positive individuals are often involved by Kaposi sarcoma. Am J Surg Pathol 2008, 32:1006-1012.

56. Kim B, Jeon YK, Kim CW: Kaposi sarcoma herpes virusassociated hemophagocytic syndrome complicated by multicentric Castleman disease and Kaposi sarcoma in a HIV-negative immunocompetent patient: an autopsy case. J Korean Med Sci 2009, 24: 970-974.

57. Wyplosz B, Carlotti A, Escaut 1, et al.: Initial human herpesvirus8 rash and multicentric Castleman disease. Clin Infect Dis 2008, 47:684-688.

58. Seliem RM, Griffith RC, Harris NL, et al.: HHV-8+, EBV+ multicentric plasmablastic microlymphoma in an HIV+ man: the spectrum of HHV-8+ lymphoproliferative disorders expands. Am J Surg Pathol 2007, 31:1439-1445.

59. Stary G, Kohrgruber N, Herneth AM, et al.: Complete regression of HIV-associated multicentric Castleman disease treated with rituximab and thalidomide. AIDS 2008, 22:1232-1234.

60. Bower M, Powles T, Williams S, et al.: Brief communication: rituximab in HIV-associated multicentric Castleman disease. Ann Intern Med 2007, 147:836-839.

61. • Gerard L, Berezne A, Galicier L, et al.: Prospective study of rituximab in chemotherapy-dependent human immunodeficiency virus associated multicentric Castleman's disease: ANRS 117 CastlemaB trial. J Clin Oncol 2007, 25:3350-3356. This article presents the first reported prospective therapeutic trial of HIVrelated MCD. The results affirm the role of rituximab as an efficacious therapeutic agent for MCD.

62. Carbone A, Gloghini A: KSHV/HHV8-associated lymphomas. $\mathrm{Br}$ J Haematol 2008, 140:13-24.

63. Boulanger E, Afonso PV, Yahiaoui Y, et al.: Human herpesvirus8 (HHV-8)-associated primary effusion lymphoma in two renal transplant recipients receiving rapamycin. Am J Transplant 2008, 8:707-710

64. Melo NC, Sales MM, Santana AN, et al.: Pleural primary effusion lymphoma in a renal transplant recipient. Am J Transplant 2008, 8:906-907.

65. Wu SJ, Hung CC, Chen $\mathrm{CH}$, et al.: Primary effusion lymphoma in three patients with chronic hepatitis B infection. J Clin Virol 2009, 44:81-83.

66. Brimo F, Michel RP, Khetani K, et al.: Primary effusion lymphoma: a series of 4 cases and review of literature with 
emphasis on cytomorphologic and immunocytochemical differential diagnosis. Cancer 2007, 111:224-233.

67. De Filippi R, Laccarino G, Frigeri F, et al.: Elevation of clonal serum free light chains in patients with HIV-negative primary effusion lymphoma (PEL) and PEL-like lymphoma. Br J Haematol 2009, 147:405-408.

68. Gasperini P, Tosato G: Targeting the mammalian target of Rapamycin to inhibit VEGF and cytokines for the treatment of primary effusion lymphoma. Leukemia 2009, 23:1867-1874.

69. Boulanger E, Meignin V, Oksenhendler E: Bortezomib (PS-341) in patients with human herpesvirus 8-associated primary effusion lymphoma. Br J Haematol 2008, 141:559-561.

70. Ripamonti D, Marini B, Rambaldi A, et al.: Treatment of primary effusion lymphoma with highly active antiviral therapy in the setting of HIV infection. AIDS 2008, 22:1236-1237.
71. Cassoni A, Ali U, Cave J, et al.: Remission after radiotherapy for a patient with chemotherapy-refractory HIV-associated primary effusion lymphoma. J Clin Oncol 2008, 26:52975299.

72. Casper C, Krantz EM, Corey L, et al.: Valganciclovir for suppression of human herpesvirus- 8 replication: a randomized, double-blind, placebo-controlled, crossover trial. J Infect Dis 2008, 198:23-30.

73. Nun TK, Kroll DJ, Oberlies NH, et al.: Development of a fluorescence-based assay to screen antiviral drugs against Kaposi's sarcoma associated herpesvirus. Mol Cancer Ther 2007, 6:2360 2370.

74. Bryant A, Milliken S: Successful reduced-intensity conditioning allogeneic HSCT for HIV-related primary effusion lymphoma. Biol Blood Marrow Transplant 2008, 14:601-602. 